\section{Early onset posterior subscapular cataract in a series of myotonic dystrophy type 2 patients}

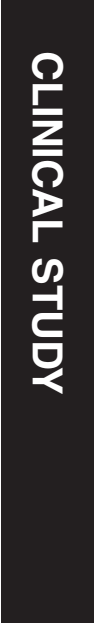

${ }^{1}$ First Department of Neurology, University of Athens, Medical School, Aeginition Hospital, Athens, Greece

${ }^{2}$ Department of Medical Genetics, University of Athens, School of Medicine, Aghia Sophia Children's Hospital, Athens, Greece

Correspondence: C Papadopoulos, First Department of Neurology, University of Athens, Medical School, Aeginition Hospital, 74, Vas. Sophias Avenue, Athens, 11528, Greece

Tel: +302107289152

Fax: +30 2107216474 .

E-mail: constantinospapado poulos@yahoo.com

Received: 25 April 2017 Accepted in revised form: 25 October 2017

Published online:

8 December 2017

\begin{abstract}
Purpose Early onset posterior subscapular cataract ( $<50$ years of age) is a characteristic feature of myotonic dystrophy type 2 (DM2). Nevertheless, despite being operated at a young age, many patients remain undiagnosed for years. The purpose of this study was to assess the prevalence of early onset posterior subscapular cataract as a presenting symptom of the disease in a cohort of patients with DM2.

Patients and methods We retrospectively reviewed medical records of DM2 patients followed in our institution for the presence of early onset posterior subscapular cataract, of any secondary causes of cataract, of the age of onset of muscle weakness and of final disease diagnosis.
\end{abstract}

Results Twenty-eight patients were studied. Nine patients $(32.1 \%)$ had presented early onset posterior subscapular cataract at a median age of 43 years (IQR $=36-46)$ and seven $(25 \%)$ reported it was the presenting sign. No patient was referred for neuromuscular evaluation due to the occurrence of early onset cataract. Median delay between cataract onset and referral for neuromuscular evaluation was $\mathbf{1 0}$ years (IQR = 6.0-19.5) and final DM2 diagnosis was achieved after a median of 16 years (IQR = 6.5-19.5).

Conclusion This study shows that early onset posterior subscapular cataract was the first symptom of the disease in $25 \%$ of our DM2 patients. Nevertheless, none was suspected of having cataract in the context of DM2, and referral for neuromuscular evaluation was made after a long delay and usually following the appearance of other symptoms. Ophthalmologists can be the first physicians encountering these patients and should have a low threshold
C Papadopoulos', K Kekou², S Xirou' S Kitsiou-Tzeli' , E Kararizou' ${ }^{1}$ and GK Papadimas ${ }^{1}$ for referring them for neuromuscular evaluation.

Eye (2018) 32, 622-625; doi:10.1038/eye.2017.280; published online 8 December 2017

\section{Introduction}

Myotonic dystrophy type 2 (DM2) or proximal myotonic myopathy (PROMM) is an autosomaldominant disorder caused by an unstable [CCTG]n tetranucleotide repeat expansion in intron 1 of the CNBP/ZNF9 gene on chromosome 3q21.3 (ref. 1). Disease onset occurs in late adulthood with a large spectrum of symptoms such as early onset cataract, myotonia, muscle stiffness and myalgia, proximal weakness, cardiac conduction defects, endocrinological abnormalities, and cognitive impairment. ${ }^{2,3}$ DM2 is probably underdiagnosed due to its large clinical variability. Some patients present with prominent myalgia and are frequently misdiagnosed as having fibromyalgia, while others present with late-onset, slowly progressive, proximal muscle weakness that is often attributed to advanced age. ${ }^{2}$ Some, finally, reach a correct diagnosis after a long period of time and usually following an extensive workout. ${ }^{4}$

Early onset posterior subscapular cataract $(<50$ years of age) is considered a characteristic feature of both myotonic dystrophy type 1 (DM1) and 2 (DM2), and at least for DM1 is known to be a key feature for timely diagnosis. ${ }^{5-7}$ In DM2 patients, early onset cataract can also be a presenting feature of the disease, preceding all other symptoms, ${ }^{8}$ making thus the ophthalmologist, sometimes, the first doctor a patient would visit. In these cases and especially in the absence of other secondary causes of cataract, ${ }^{9}$ awareness by the treating physician that early onset cataract can be a presenting manifestation of a multisystemic 
Table 1 Patients' main characteristics

\begin{tabular}{|c|c|c|c|c|c|c|}
\hline & Sex/Age & $\begin{array}{c}\text { Age at cataract } \\
\text { diagnosis }\end{array}$ & $\begin{array}{c}\text { Age at weakness } \\
\text { presentation }\end{array}$ & $\begin{array}{c}\text { Age at neuromuscular } \\
\text { evaluation }\end{array}$ & $\begin{array}{c}\text { Reason for neuromuscular } \\
\text { evaluation }\end{array}$ & $\begin{array}{l}\text { Age at final } \\
\text { diagnosis }\end{array}$ \\
\hline 1. & $\mathrm{~F} / 72$ & No & 24 & 59 & Muscle weakness & 60 \\
\hline 2. & $\mathrm{M} / 64$ & 57 & 55 & 57 & Muscle weakness & 59 \\
\hline 3. & $\mathrm{~F} / 80$ & 69 & 60 & 68 & Muscle weakness & 69 \\
\hline 4. & $\mathrm{~F} / 46$ & No & No & 35 & Diagnosis in a relative & 35 \\
\hline 5. & $\mathrm{~F} / 67$ & 55 & 55 & 60 & Muscle weakness & 60 \\
\hline 6. & $\mathrm{~F} / 66$ & 43 & 46 & 53 & Muscle weakness & 53 \\
\hline 7. & $\mathrm{M} / 70$ & 48 & 65 & 68 & Muscle weakness & 68 \\
\hline 8. & $\mathrm{M} / 74$ & 70 & 71 & 72 & Muscle weakness & 72 \\
\hline 9. & $\mathrm{M} / 68$ & 55 & No & 62 & HyperCKemia & 63 \\
\hline 10. & $\mathrm{~F} / 44$ & No & No & 36 & Diagnosis in a relative & 36 \\
\hline 11. & $\mathrm{~F} / 69$ & 41 & 40 & 60 & Muscle weakness & 60 \\
\hline 12. & $\mathrm{~F} / 68$ & No & 61 & 65 & Muscle weakness & 66 \\
\hline 13. & $\mathrm{~F} / 64$ & 46 & 42 & 53 & Muscle weakness & 54 \\
\hline 15. & $\mathrm{M} / 50$ & 35 & 40 & 40 & Muscle weakness & 40 \\
\hline 16. & $\mathrm{~F} / 67$ & No & 43 & 53 & Myotonia & 53 \\
\hline 17. & $\mathrm{~F} / 68$ & 46 & 60 & 63 & Muscle weakness & 63 \\
\hline 18. & $\mathrm{~F} / 71$ & No & 68 & 72 & Muscle weakness & 72 \\
\hline 19. & $\mathrm{~F} / 42$ & No & No & 41 & Myotonia & 41 \\
\hline 20. & $\mathrm{M} / 62$ & No & 45 & 58 & Muscle weakness & 58 \\
\hline 21. & $\mathrm{M} / 37$ & No & 20 & 32 & Myotonia & 32 \\
\hline 22. & $\mathrm{~F} / 73$ & 25 & 64 & 70 & Muscle weakness & 70 \\
\hline 23. & $\mathrm{M} / 41$ & 37 & No & 38 & HyperCKemia & 38 \\
\hline 24. & $\mathrm{~F} / 63$ & 45 & 50 & 52 & Muscle weakness & 61 \\
\hline 25. & $\mathrm{M} / 29$ & No & 22 & 26 & Muscle weakness & 26 \\
\hline 26. & $\mathrm{M} / 54$ & No & 50 & 50 & HyperCKemia & 50 \\
\hline 27. & $\mathrm{~F} / 71$ & 70 & 60 & 62 & Muscle weakness & 62 \\
\hline 28. & $\mathrm{~F} / 79$ & No & 48 & 53 & Muscle weakness & 69 \\
\hline
\end{tabular}

disease is essential for appropriate referral of patients, avoidance of unnecessary examinations and timely diagnosis. Nevertheless, to our experience, many patients with DM2 despite being diagnosed and operated at a young age for posterior subscapular cataract, remain undiagnosed for years and are referred for evaluation at a neuromuscular unit only after appearance of muscle weakness or another symptom of the disease.

The objectives of our study were to (1) assess the prevalence of early onset posterior subscapular cataract as a presenting symptom of the disease, (2) assess the diagnostic delay between cataract diagnosis and diagnosis of the underlying disease, in a cohort of patients with genetically confirmed DM2, in order to increase awareness of treating physicians and especially ophthalmologists for the association of early onset cataract and this multisystemic disorder.

\section{Materials and methods}

We retrospectively included all patients with a genetically confirmed diagnosis of DM2 admitted in our institution between January 2003 and 2017. The diagnosis was made in patients referred for evaluation with the following symptoms or signs: muscle weakness and/or atrophy, high creatine kinase (CK) levels, known family history of the disease. Written informed consent was obtained from all recruited patients when primary diagnostic procedures were performed, with explicit consent for future use for research purposes, according to the Declaration of Helsinki and the study was approved by our local Ethics Committee.

Patients' medical records were reviewed and any information regarding the presence of early onset posterior subscapular cataract (cataract occurring at an age of 50 years or less), the presence of any cause of secondary cataract, the age of onset of muscle weakness and the age of disease diagnosis was entered in a dedicated database. Data are presented as medians and interquartile ranges (IQR) for continuous variables, and as counts and percentages (\%) for discrete variables.

\section{Results}

We identified a cohort of 28 patients (11 men) with a diagnosis of DM2 followed in our institution. Patients' main characteristics are presented in Table 1. Among them nine patients $(32.1 \%)$ had presented early onset posterior subscapular cataract at a median age of 43 years $(\mathrm{IQR}=36-46)(\min =25 ; \max =48)$. Two patients already 
reported muscle weakness at the time of cataract diagnosis, while seven $(25 \%)$ reported that it was the presenting sign of the disease. One patient was under treatment for type 2 diabetes at the time cataract was diagnosed. Two patients underwent a muscle biopsy in the framework of muscle weakness due to failure to recognize cataract as an integral symptom of their disease. No patient was referred for neuromuscular evaluation due to the occurrence of early onset cataract. Twenty patients were referred for evaluation of muscle weakness, three for evaluation of asymptomatic hyperCKemia, three for evaluation of myotonia, and two following diagnosis of the disease in another family member. Median delay between cataract onset and referral for evaluation at a specialized neuromuscular unit was 10 years $(\mathrm{IQR}=6.0$ 19.5). Final DM2 diagnosis was achieved after a median of 16 years $(\mathrm{IQR}=6.5-19.5)(\min =1 ; \max =45)$ following cataract diagnosis.

\section{Discussion}

In this study, we show that early onset posterior subscapular cataract was the first reported symptom in $25 \%$ of DM2 patients followed in our institution. Nevertheless, and despite the absence of alternative causes of early onset cataract, none of our patients was suspected of having cataract in the context of a multisystemic disorder, notably DM2, and referral for neuromuscular evaluation was made after a long delay and usually following the appearance of other symptoms of the disease.

DM2 was first described in Europe and in the United States as a proximal myotonic myopathy or a myotonic dystrophy without CTG expansion ${ }^{10-13}$ and in 2001 it was demonstrated that is caused by a CCTG expansion in intron 1 of the zinc finger protein 9 (ZNF9) gene. ${ }^{14}$ Since these original descriptions, it became evident that early onset posterior subscapular cataract was part of the clinical spectrum and one of the core symptoms of the disease. ${ }^{10,11,15}$ The incidence of posterior subcapsular cataract in DM2 ranges from 36 to $78 \%$, increasing with age. It can be present as early as the second decade of life, with a reported age of extraction ranging from 28 to 74 years and is the initial feature of the disease in 5 to $8 \%$ of patients. ${ }^{3,4,16}$ In our series, early onset posterior subscapular cataract was present in $9 / 28$ of patients $(32.1 \%)$ and was reported to be the first symptom of the disease in 7 (25\%). We believe that the higher than previously reported frequency of cataract as the presenting symptom of the disease in our series, could be probably due to a recall bias of other symptoms, such as muscle stiffness or fatigue, that are more subtle and less debilitating than muscle weakness and can be underreported by patients. These seven patients did not report any muscle weakness or symptoms severe enough, to interfere with everyday activities and to warrant evaluation by a physician prior to cataract occurrence. Even if it is not clear if cataract was indeed the first symptom of the disease, it was, however, the one that brought these patients to medical consultation for the first time. Despite the absence of any cause of secondary cataract in the vast majority of these patients, a myotonic syndrome was not considered in the differential diagnosis and neuromuscular evaluation was only performed after appearance of other symptoms of the disease, such as muscle weakness, myotonia, or following diagnosis in another member of the family. This resulted in a long delay for referral and final diagnosis reaching a median of 10 and 16 years, respectively. A long delay between symptom onset and DM2 final diagnosis has already been reported with an average delay of final diagnosis of 14 years and of $10.1( \pm 9.1)$ years when cataract is the first symptom, ${ }^{4}$ due to failure to recognize it as one of the core symptoms of the disease. ${ }^{8}$ Patients with early onset subscapular cataract should specifically be questioned by treating physicians about subtle symptoms of DM2, such muscle stiffness and pain, fatigue, difficulties rising from a chair or climbing stairs and about the presence of family history of weakness or of a multisystemic disorder.

Early onset posterior subscapular cataract is common among DM1 patients and is considered a key finding for early diagnosis of the disease. ${ }^{6,7}$ Screening for DM1 is recommended for patients under 55 years of age, with bilateral cataracts and no other precipitating factors. ${ }^{17}$ To our knowledge, similar studies about the prevalence of DM2 mutation in cataract patients have not been performed to date, but given the high occurrence of cataract as an early symptom of the disease reported by us and others, ${ }^{3,4}$ it would be useful to do so, in order to establish appropriate guidelines about timely genetic testing. A lower threshold for genetic testing in these patients would probably facilitate diagnosis and decrease diagnostic delay and could prevent from performing unnecessary tests, such as the muscle biopsies preformed in two of our patients in the framework of muscle weakness, because of failure to integrate early onset cataract in the context of a neuromuscular disorder. Finally, an early genetic diagnosis of DM2 in these cases will also facilitate appropriate follow-up of the multisystemic clinical features of the disease, such as diabetes and cardiac arrhythmias, improving patients' prognosis.

In conclusion, in this series of DM2 patients, early onset posterior subscapular cataract was the first symptom of the disease warranting medical attention in $23 \%$ of patients. Nevertheless, in no patient was a myotonic syndrome suspected because of the presence of early onset cataract and final diagnosis was only made after a 
long delay and following appearance of other symptoms of the disease. Ophthalmologists can be the first physicians that encounter these patients and should be aware that early onset posterior subscapular cataract can be the presenting feature of DM2. They should specifically ask about other subtle signs of the disease and finally, have a low threshold for referring these patients for neuromuscular evaluation.

\section{Summary}

\section{What was known before}

- Early onset posterior subscapular cataract ( $<50$ years of age) is considered a characteristic feature of both myotonic dystrophy type 2, sometimes preceding all other symptoms. Nevertheless, to our experience, many patients despite being diagnosed and operated at a young age for posterior subscapular cataract are only referred for evaluation at a neuromuscular unit after appearance of muscle weakness or another symptom of the disease.

\section{What this study adds}

- In this cohort of Greek patients with genetically confirmed myotonic dystrophy type 2, we show that early onset posterior subscapular cataract was the first reported symptom in $25 \%$ of patients. Despite the absence of alternative causes of early onset cataract, none was suspected of having cataract in the context of a multisystemic disorder, notably myotonic dystrophy type 2 , and referral for neuromuscular evaluation was made after a long delay. Ophthalmologists can be the first physicians that encounter these patients, should specifically ask about subtle signs of the disease and should have a low threshold for referring them for neuromuscular evaluation.

\section{Conflict of interest}

The authors declare no conflict of interest.

\section{References}

1 Finsterer J. Myotonic dystrophy. Eur J Neurol 2002; 9: 441-447.

2 Meola G. Clinical aspects, molecular pathomechanisms and management of myotonic dystrophies. Acta Myol 2013; 32(3): 154-165.

3 Day JW, Ricker K, Jacobsen JF, Rasmussen LJ, Dick KA, Kress W et al. Myotonic dystrophy type 2: molecular, diagnostic and clinical spectrum. Neurology 2003; 60(4): 657-664.
4 Hilbert JE, Ashizawa T, Day JW, Luebbe EA, Martens WB, McDermott MP et al. Diagnostic odyssey of patients with myotonic dystrophy. J Neurol 2013; 260(10): 2497-2504.

5 Turner C, Hilton-Jones D. The myotonic dystrophies: diagnosis and management. J Neurol Neurosurg Psychiatry 2010; 81: 358-367.

6 Voermans NC, Erasmus CE, Ockeloen CW, Van Engelen BG, Eggink CA. Primary cataract as a key to recognition of myotonic dystrophy type 1. Eur J Ophthalmol 2015; 25(4): e46-e49.

7 Medica I, Teran N, Volk M, Pfeifer V, Ladavac E, Peterlin B. Patients with primary cataract as a genetic pool of DMPK protomutation. J Hum Genet 2007; 52: 123-128.

8 Udd B, Meola G, Krahe R, Thornton C, Ranum L, Day J et al. Report of the 115th ENMC workshop: DM2/PROMM and other myotonic dystrophies. 3rd Workshop, 14-16 February 2003, Naarden, The Netherlands. Neuromuscul Disord 2003; 13(7-8): 589-596.

9 Gupta VB, Rajagopala M, Ravishankar B. Etiopathogenesis of cataract: an appraisal. Indian J Ophthalmol 2014; 62(2): 103-110.

10 Ricker K, Koch MC, Lehmann-Horn F, Pongratz D, Otto M, Heine R et al. Proximal myotonic myopathy: a new dominant disorder with myotonia, muscle weakness, and cataracts. Neurology 1994; 44: 1448-1452.

11 Udd B, Krahe R, Wallgren-Petterson C, Falck B, Kalimo H. Proximal myotonic dystrophy: a family with autosomal dominant muscular dystrophy, cataracts, hearing loss and hypogonadism: heterogeneity of proximal myotonic syndromes? Neuromuscul Disord 1997; 7: 217-228.

12 Thornton CA, Griggs RC, Moxley RT. Myotonic dystrophy with no trinucleotide repeat expansion. Ann Neurol 1994; 35: 269-272.

13 Day JW, Roelofs R, Leroy B, Pech I, Benzow K, Ranum LPW. Clinical and genetic characteristics of a five-generation family with a novel form of myotonic dystrophy (DM2). Neuromuscul Disord 1999; 9: 19-27.

14 Liquori C, Ricker K, Moseley ML, Jacobsen JF, Kress W, Naylor SL et al. Myotonic dystrophy type 2 caused by a CCTG expansion in intron 1 of ZNF9. Science 2001; 293: 864-867.

15 Moxley RT, Udd B, Ricker K. Proximal myotonic myopathy (PROMM) and other proximal myotonic syndromes. Neuromuscular Disord 1998; 8: 519-520.

16 Dalton JC, Ranum LPW, Day JW. Myotonic dystrophy type 2. In: Pagon RA, Adam MP, Ardinger $\mathrm{HH}$, Wallace SE, Amemiya A, Bean LJH, Bird TD, Ledbetter N, Mefford HC, Smith RJH, Stephens Keditors (eds). GeneReviews ${ }^{\circledR}$ [Internet], University of Washington, Seattle: Seattle, WA, 2006, pp 1993-2017.

17 Kidd A, Tumpenny P, Kelly K, Clark C, Church W, Hutchinson $\mathrm{C}$ et al. Ascertainment of myotonic dystrophy through cataract by selective screening. J Med Genet 1995; 32: 519-523. 